\title{
Posttraumatic Biloma formation: \\ DIAGNOSIS AND MANAGEMENT
}

\author{
Ricardo Vieira Teles Filho1, Guilherme de Matos $\mathrm{Abe}^{2}$
}

Clin Biomed Res. 2019;39(3):255-256

1 Departamento de Radiologia e Diagnóstico em Imagem, Faculdade de Medicina, Universidade Federal de Goiás (UFG). Goiânia, GO, Brasil.

2 Faculdade de Medicina, Universidade Federal de Goiás (UFG). Goiânia, GO, Brasil.

Corresponding author: Ricardo Vieira Teles Filho ricardovteles@gmail.com Faculdade de Medicina, Universidade Federal de Goiás (UFG)

Avenida dos Alpes, Quadra 49, Lote 11, Setor União, 400.

74313-760, Goiânia, GO, Brasil.

\begin{abstract}
One of the rarest complications of the hepatic trauma is a biloma, defined as an abnormal bile collection outside the biliary tree, with intra or extrahepatic localization. Patients with biloma do not present with specific clinical features, which demands a challenging radiological diagnosis. In this report, we present a case of biloma due to blunt hepatic trauma, in which the patient experienced a changing symptomatic spectrum after surgery and had an interesting radiological investigation. The clinical course, imaging features, and management of this case are discussed.
\end{abstract}

Keywords: Biloma; biliary leak; liver trauma

A 25-year-old male patient sustained complex blunt hepatic trauma after abdominal crush injury by an object heavier than $100 \mathrm{~kg}$. He had liver laceration, pulmonary contusion and hemothorax. Exploratory laparotomy was performed, followed by urgent hepatorrhaphy. After 3 weeks, the patient presented with sharp pain and discomfort in the upper right quadrant and abdominal distension associated with intense nausea. An abdominal ultrasound (US) demonstrated voluminous liver cyst formation. Abdominal computed tomography (CT) was performed and showed voluminous intrahepatic loculated fluid collection (Figure 1). Magnetic resonance cholangiopancreatography (MRCP) was then performed (Figure 2) to exclude cystic lesion and to establish the actual location of the lesion; it demonstrated extra- and intrahepatic biliary tree injury (Figure 3 ). Abdominal drainage of the collection was performed by ultrasound-guided percutaneous drainage, collecting about $1,500 \mathrm{~mL}$ of bile, and confirmed the diagnosis of biloma. The procedure had no complications, and the patient was discharged with an abdominal tube draining biliary secretion, which was removed after 3 weeks. After two months, follow-up CT demonstrated the resolution of the case. The patient was then followed every two months for the following six months after the drainage procedure, with tomographic studies demonstrating no new biliary leak. Hence, he was finally discharged in good medical condition. 


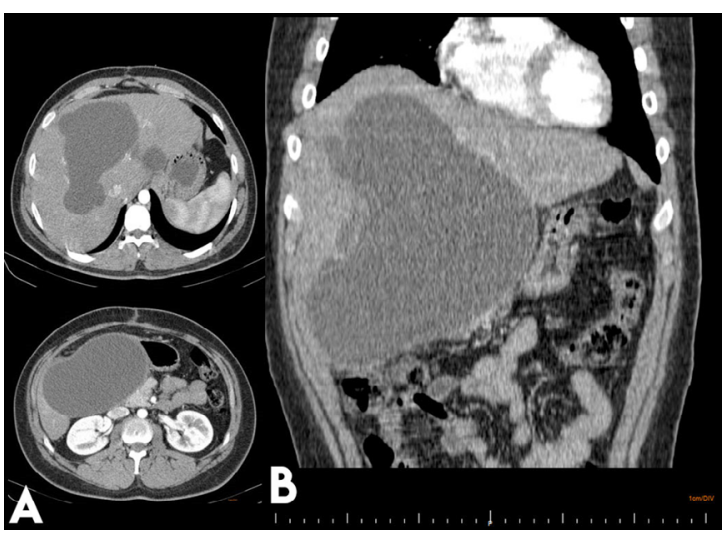

Figure 1: (A)Axial contrast-enhanced computed tomography (CT) of the liver taken 6 weeks after the injury demonstrating intrahepatic loculated fluid collection. Drainage of this collection confirmed the presence of biloma. (B) Coronal reconstructed contrast-enhanced $C T$ of the same site in the liver also demonstrating intrahepatic loculated fluid collection.
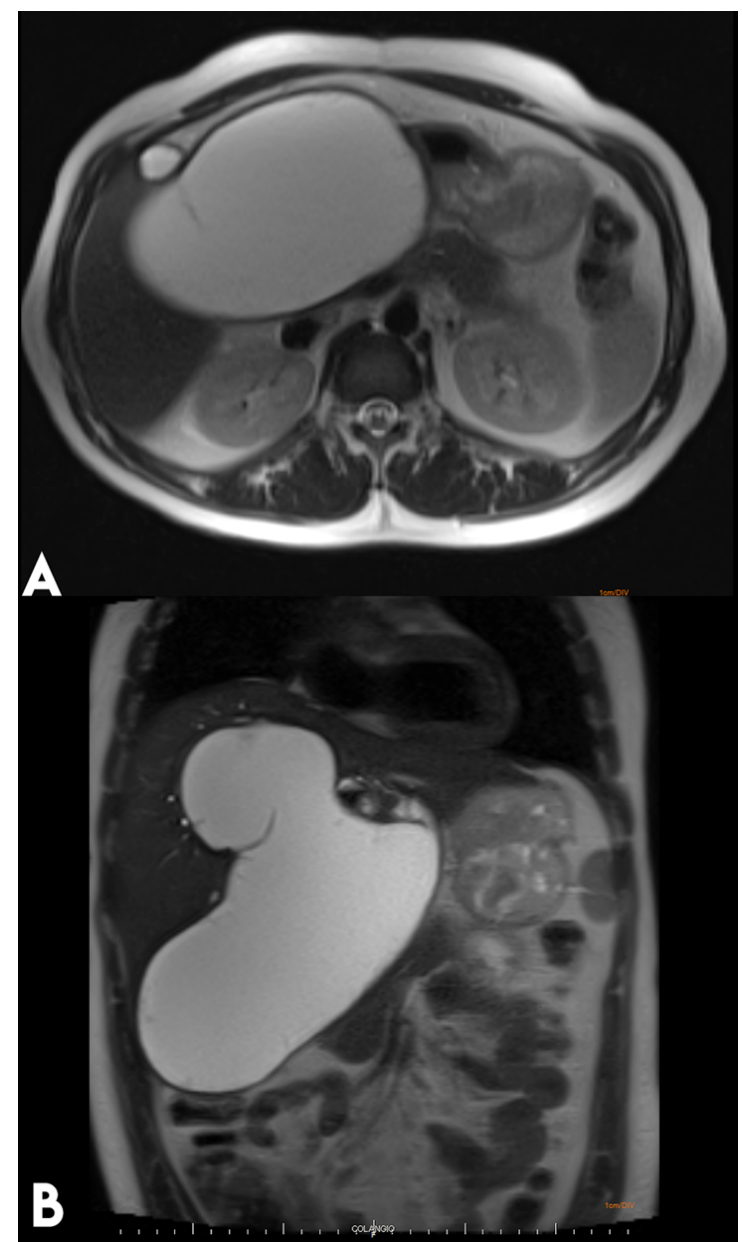

Figure 2: (A) Axial T2-weighted magnetic resonance (MR) image showing fluid collection. T2-weighted sequences produce bright signal in stationary fluids. (B) Coronal T2weighted MR image showing fluid collection. T2-weighted sequences also produce bright signal in stationary fluids.
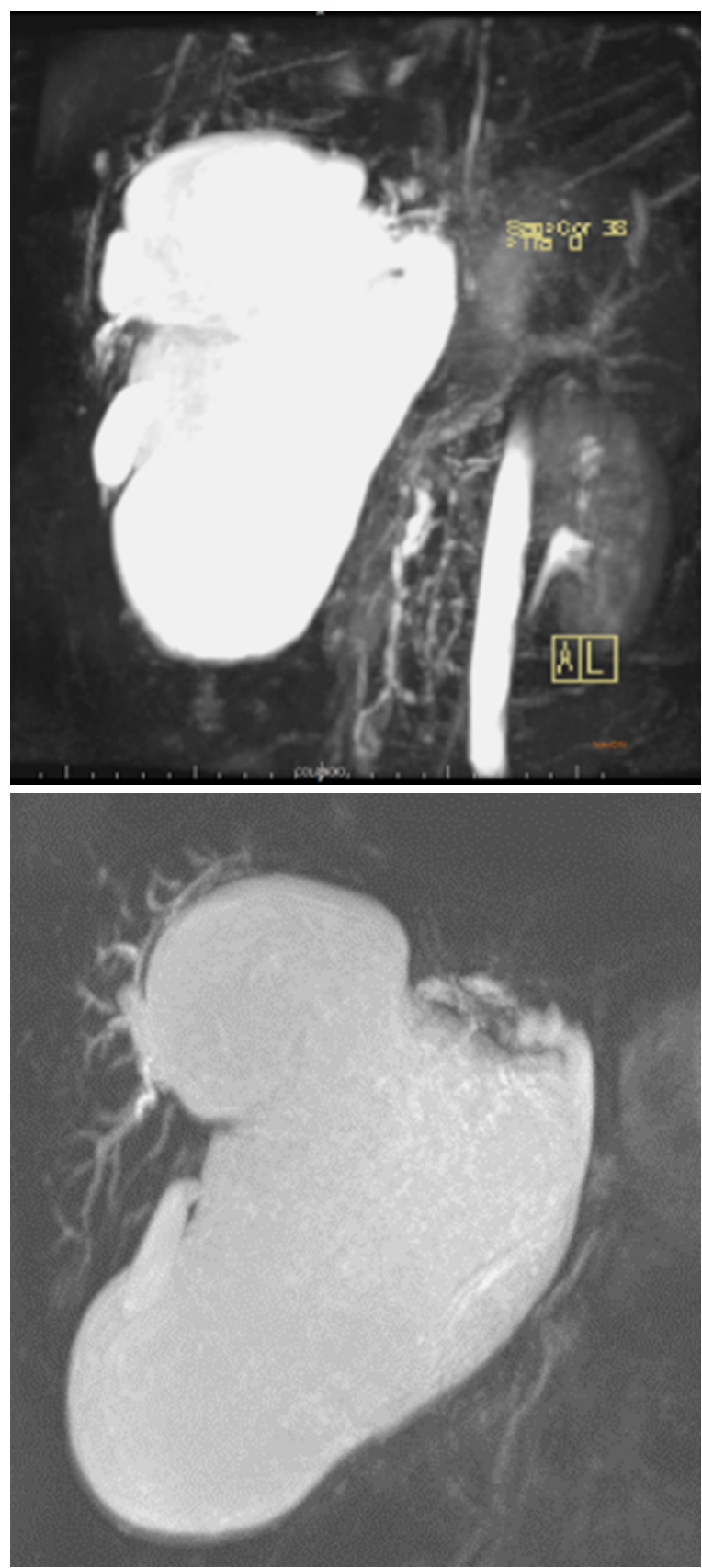

Figure 3: Coronal magnetic resonance (MR) image using maximum intensity projection (MIP) shows intrahepatic bile duct dilatation. Images demonstrate intra- and extrahepatic hyperintense collection. The collection resolved completely after ultrasound-guided percutaneous puncture.

\section{DISCUSSION}

Biloma is the collection of bile, encapsulated or not, outside the biliary tree, either inside or outside the liver. Etiologies are spontaneous biloma, injury (blunt or penetrating), and iatrogenic injury to the biliary tree ${ }^{1}$. The gallbladder is the most common site of biliary injury in blunt trauma, followed by the extra- and intrahepatic biliary ducts, respectively. 
The average time between symptom onset and diagnosis is one to two weeks. Posttraumatic intrahepatic biliary cysts, or bilomas, were first described in 1898 by Whipple, in a patient hit by a horse. Since then, few reports of bilomas have been published ${ }^{2}$.

The detection of biliary tree injury during surgical treatment of blunt hepatic trauma is challenging. Therefore, for a surgeon, a biloma is a disease to be considered, and a high level of suspicion is required during the follow-up of blunt hepatic trauma. A delayed diagnosis of biloma due to biliary tree injury usually results in increased morbidity. A US is usually used as the first exam to detect a biloma. However, a CT scan is more effective in determining the collection's relation to adjacent structures. Differential diagnosis must be performed and includes cysts, seromas, pseudocysts, hematomas and liver abscesses. MRCP offers a noninvasive method for obtaining images of the biliary system, being useful to establish if the lesion is intra- or extrahepatic, to only then proceed with proper treatment ${ }^{3}$.
Guided percutaneous puncture, in this case using US, helps in the diagnosis and treatment. For this reason, percutaneous drainage has been proposed as first therapeutic option; the success rate of the procedure is $80 \%{ }^{4}$. If there is no resolution, the interventional radiologist may opt to perform endoscopic retrograde cholangiopancreatography and sphincterotomy, with stent placement or not. Long-term follow-up of these patients is necessary due to a possible new complication of the trauma of the biliary tract, which is ductal stenosis, with consequent recurrent cholangitis and hepatic cirrhosis ${ }^{5}$.

\section{Conflict of interest}

Authors declare that they have no conflict of interest. All procedures performed in studies involving human participants were in accordance with the ethical standards of the institutional and/or national research committee and with the 1964 Helsinki declaration and its later amendments or comparable ethical standards.

\section{REFERENCES}

1. Copelan A, Bahoura L, Tardy F Kirsch M, Sokhandon F, Kapoor B. Etiology, Diagnosis, and management of bilomas: a current update. Tech Vasc Interv Radiol. 2015;18(4):236-43.

2. Hashemi SR, Ghaemian N, Marzbali NA, Mohammadhasani AR. Biloma due to blunt liver trauma. Casp J Intern Med. 2010;1(4):159-61.
3. Esensten M, Ralls PW, Colletti P, Halls J. Posttraumatic intrahepatic biloma: sonographic diagnosis. AJR Am J Roentgenol. 1983;140(2):303-5

4. Melamud K, LeBedis CA, Anderson SW, Soto JA. Biliary imaging: multimodality approach to imaging of biliary injuries and their complications. Radiographics. 2014;34(3):613-23.
5. Thomas S, Jahangir K. Noninvasive Imaging of the biliary system relevant to percutaneous interventions. Semin Intervent Radiol. 2016;33(4):277-82.

Received: July 2, 2019 Accepted: Aug 1, 2019 